\title{
BLOOD SUPPLY OF THE OPTIC NERVES AND CHIASMA AND ITS CLINICAL SIGNIFICANCE*
}

\author{
BY \\ BRODIE HUGHES \\ Professor of Neurosurgery, University of Birmingham
}

\section{Part I The Optic Nerve}

The blood supply of the optic nerve has been a subject of controversy for at least 200 years, since the description of the arterial circle around the optic nerve head by Zinn (1755) and Haller (1754). Numerous papers have been written concerning the subject and in the past few years new techniques of injection, micro-angiography, and dissection and Neoprene casts have resulted in further papers on the subject. Older writers, using relatively crude techniques, differed widely in their interpretation of the blood supply of the nerve. What is, perhaps, more surprising is that modern workers using highly elaborate and skilled techniques have also differed considerably in their descriptions of the vascular supply. The differences are especially noteworthy when considering the central vessels of the optic nerve, the anastomoses around the optic nerve head, and the blood supply of the intracranial portion of the optic nerve.

All observers are agreed that the optic nerve is surrounded by a pial plexus of vessels and also that it contains some system of central vessels. There is disagreement concerning the contribution made to these systems by individual vessels and the degree of anastomosis between them.

Most workers seem to be agreed that the outer layers of the optic nerve in its orbital portion are nourished from a capillary pial plexus. This is contributed to mainly by twigs from the ophthalmic artery, and its branches the long and short posterior ciliary arteries. François and Neetens (1954) postulate also twigs from the lacrimal artery. How much of the nerve is supplied by this pial plexus seems to be a matter of speculation. Steele and Blunt (1956) and many other writers have noted that branches from this plexus appear to penetrate the optic nerve for short distances only, so that it may be inferred that probably less than half the fibres are supplied from this source and that they are those situated peripherally in the nerve. There seems to be no convincing evidence from these studies that there is any horizontal or vertical division of blood supply in this peripheral portion. The only other point at issue over the pial plexus is the contribution from the central artery itself. All writers other than François and Neetens agree that this vessel

* Read in abridged form at the combined meeting of the Society of British Neurological Surgeons and the 36th Annual Meeting of the Irish Ophthalmological Society in Dublin, May 16-18, 1957 
gives off branches to the pial plexus before its entry into the optic nerve, so that it may be assumed that in most cases some local contribution, presumably to the inferior peripheral fibres, derives from the central artery outside the nerve.

The main controversy concerns the origin, distribution, and method of branching of the central vessels. Branches have been described from the central artery itself within the nerve (Steele and Blunt, 1956; Wybar, 1956). A central artery of the optic nerve itself has been described arising from the ophthalmic artery (François and Neetens, 1954), and many authors have described anterior and posterior axial vessels in the nerve derived from the central artery either before or after its entry into the optic nerve. Though many workers have denied the presence of any branches from the central retinal artery within the optic nerve, no one who has studied the excellent illustrations of Steele and Blunt (1956) and of Wybar (1956) can deny their existence. In what proportion of subjects these branches exist must remain a matter of speculation and is not of great importance.

Most workers have found axial vessels entering the optic nerve with the central retinal artery and arising from it just before its entry. In some cases the origin may be after the vessel has entered the nerve and in some cases its origin may be far back and may arise from the ophthalmic artery itself rather than from the central retinal artery (François and Neetens, 1954). The distribution of the axial vessels, variously termed the collateral arteries of the central retinal artery, anterior and posterior axial arteries, or central artery of the optic nerve, has also been the subject of controversy. Earlier writers tended to adhere to the description of the axial vessels running forward and backward throughout most of the length of the orbital optic nerve, and such a vessel was described by Vossius (1883). Since then varying descriptions have been given, and it seems commoner to find a long branch directed posteriorly as far as the optic canal than a long anterior branch (Wolff, 1948). Others, notably Wybar (1956), whilst demonstrating the presence of axial branches, deem them to be only short vessels with a limited area of supply.

To collate information on this point, therefore, it seems probable that the central core of the optic nerve within the orbit is supplied in a variety of ways. Contributions may come from the intraneural part of the central artery of the retina, from axial vessels derived from the central artery before its entry into the optic nerve which may send out anterior and posterior axial branches, and sometimes from a separate central artery of the optic nerve arising from the ophthalmic artery in the orbit. All such systems have been described by highly skilled and competent observers and must be accepted as present in the specimens they dissected. The variability in supply must merely reflect the vagaries of human anatomy, and the only fault lies in our own scientific or Procrustean attempts to cover in a single rigid description a system of considerable variation. 
The same situation appears at the optic nerve head. Here the pial plexus receives contributions from the circle of Zinn, and the point at issue is to what degree, if any, arterial anastomosis takes place between the choroidal and optic nerve arterial supply, for all are agreed that the capillary anastomosis seems to be present. The answer can only be that such anastomosis is present in some cases but probably not in most.

The blood supply of the intracranial optic nerve has also been subject to considerable difference of opinion. Dawson (1948) described and illustrated a sizeable branch of the ophthalmic artery running along the infero-medial aspect of this portion of the nerve and giving off branches to it. Steele and Blunt (1956) have denied this and state that, in 34 dissections in which this vessel was especially searched for, it was found in none. On the other hand, they assert that the major supply to this area of the nerve is from a branch of the anterior superior hypophyseal artery running along the under side of the nerve. They did find small branches from the ophthalmic artery going to the nerve, but these were very small and only found in the region of the optic canal. Other writers have noted contributions from the anterior cerebral, anterior communicating, and internal carotid arteries. We must be faced here with a similar situation and can only conclude that all the vessels named do, on occasion, contribute to the blood supply of this portion of the optic nerve, but that the individual variations are much greater than one commonly supposes. My own observations during operations on this region add yet another possibility. I have frequently noted a considerable contribution to the upper surface of the optic nerve from the ophthalmic artery and from the anterior cerebral artery. Both these vessels are relatively large, and easily seen by the naked eye during surgical operations, and have been observed a great many times. Viewing a vessel on the surface of the nerve does not necessarily imply that it supplies this area of the nerve but such would seem probable.

Of the arteriolar pattern relatively little has been said. Most of the supplying vessels are small and on entry into the nerve break up into small arterioles. Perhaps little more than this can usefully be said. Capillary patterns have been studied with great care by François, Neetens, and Collette (1955), and François and Neetens (1956). They have described, in the orbital portion of the optic nerve, a system of capillaries surrounding fibre bundles and appearing in transverse sections as pentagonal boxes surrounding these bundles, whilst in longitudinal sections these circles are connected by longitudinally running capillaries and thus the appearance is one of long rectangles. In the intracranial portion of the optic nerve, this regular pattern is soon lost and gives way to the more irregular meshworks of the chiasma.

Certain inferences can be drawn from this pattern of vascularization which may have clinical significance. Firstly, there seems to be a clear distinction in the orbital portion of the optic nerve between peripheral and central 
supply. The peripheral fibres are supplied from a rich pial plexus to which many vessels may contribute. The central area, by contrast, appears to be supplied by fewer vessels and perhaps on occasion by one only, the central artery of the optic nerve or the collateral artery of the optic nerve. Disturbance of function from a vascular cause may well show, therefore, predominantly peripheral or central field loss. Again, one might imagine that the central area could be more vulnerable than the peripheral because a single small lesion, compressing, clotting, or otherwise interfering with blood flow in a single small vessel, could inhibit or destroy function in the whole of the central area of this nerve segment. Secondly, apart from this centralperipheral separation, there is no evidence in the arterial, arteriolar, capillary, or venous patterns to suggest segmentation of another kind. Thirdly, capillary patterns at least suggest that they are based on bundles of fibres and therefore the effects of ischaemia should be recognizable in field loss of a "nerve fibre bundle" type. Fourthly, in the intracranial portion of the optic nerve, the central-peripheral segregation and the fibre bundle capillary box disappear, so that one would expect these features of optic nerve lesions to disappear also. On the other hand, the blood supply in this region appears to be, in some cases at least, much more dependent on single vessels. Steele and Blunt found in their dissections that the anterior superior hypophyseal artery was so often the sole vessel of supply that it might conceivably be called the vessel of the intracranial optic nerve. Where this is not so it may well be replaced by another single vessel, the pre-chiasmal artery of Dawson. In these circumstances one might infer that this portion of the optic nerve was especially vulnerable to pressure from below and medially, or to any arterial disease that might affect either of these vessels.

The clinical aspects of the subject may now be discussed and the effects on optic nerve function of vascular disease, pressure, trauma, and so forth may be examined to see if they accord with the principles inferred from anatomy.

Practitioners of clinical perimetry are accustomed to recognize at least two stigmata of vascular disease in the optic nerve, fibre bundle defects and horizontal hemianopias, usually in the lower field. It may be as well to consider a group of vascular lesions to determine how justified this assumption may be.

The hemianopic theory probably originated from those peculiar cases in which vision is lost after massive haemorrhage, often gastric in origin. When vision and field are recovered or preserved, the field pattern is characteristically that of an inferior, or less commonly superior, hemianopia. The line of demarcation lies strictly along the horizontal meridian and it is usual to find in addition generalized depression in the preserved field. No one seems to have determined whether this lesion lies in the optic nerve or the retina, certainly the horizontal line of demarcation would suggest a retinal origin. In some cases, however, the picture is not so clear cut. In one example the fundus was observed throughout the episode and the retinal 
vessels were noted to be normal. In this case the line of demarcation did not follow exactly the horizontal meridian and it seems probable that the lesion lay in the optic nerve. Another and clearer example is the following:

A young man suffered from angiomatosis of the optic nerves, some portion of the angioma being visible on the optic nerve head. He was subject to periodic attacks of inferior hemianopia in either eye lasting about half an hour. On one occasion the hemianopia in one eye failed to recover as usual and left a permanent inferior hemianopia with clear-cut margin along the horizontal meridian. The retinal vascular tree during this time was noted to be quite normal.

A not dissimilar picture may be seen in the so-called arterio-sclerotic atrophy of the optic nerve, though in this instance the evolution may be a gradual one. A similar example has been seen in association with arterial hypertension; in this instance the onset of the hemianopia was sudden and had all the characteristics of an arterial thrombosis (Fig. 1).

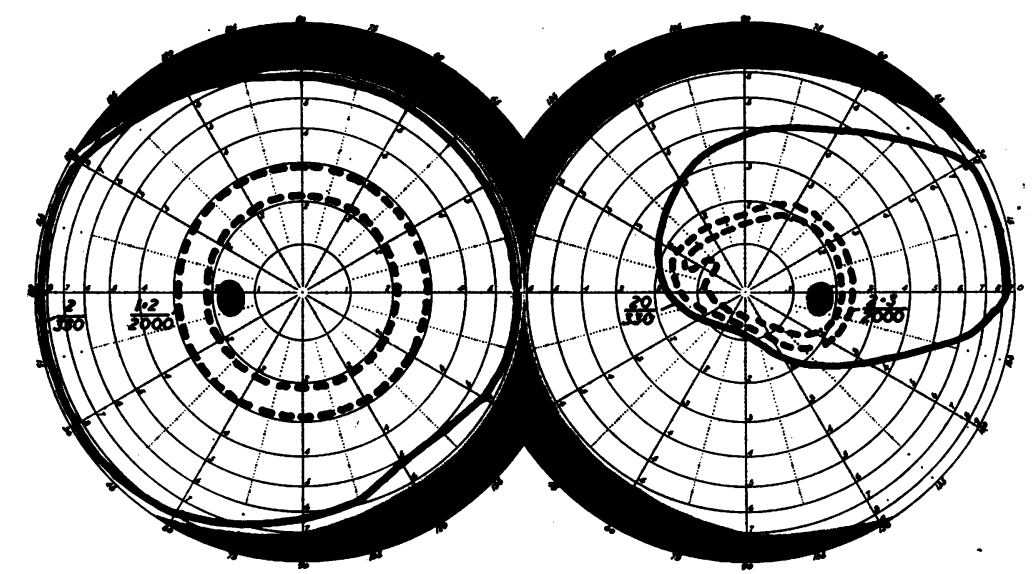

FIG. 1.-Visual field in a case of hypertension with probable arterial thrombosis in right optic nerve. Sudden onset of field loss without detectable change in retinal vessels.

These are but a few examples from a vast store of material in the literature and in personal records. Such clinical experience suggests rather strongly that there is some segmental arterial supply in the optic nerves whereby the blood supply of one horizontal half of an optic nerve may be cut off by a single lesion. In many of such cases the central area of the field is spared, but in some this is also affected. None of the anatomical work reported has provided any explanation of this clinical experience.

Another fact which has emerged clearly from anatomical work is that the central and peripheral areas of the optic nerve have a separate blood supply, though variations are considerable and there may be some anastomosis. In such conditions one might expect to find separate examples of both central and peripheral field loss in vascular lesions, and, in my experience, such is the case. The commonest type of field loss in so-called arterio-sclerotic optic atrophy is the appearance of multiple indentations in the peripheral field. 
These most commonly affect the lower field but are also seen in the upper field (Fig. 2).

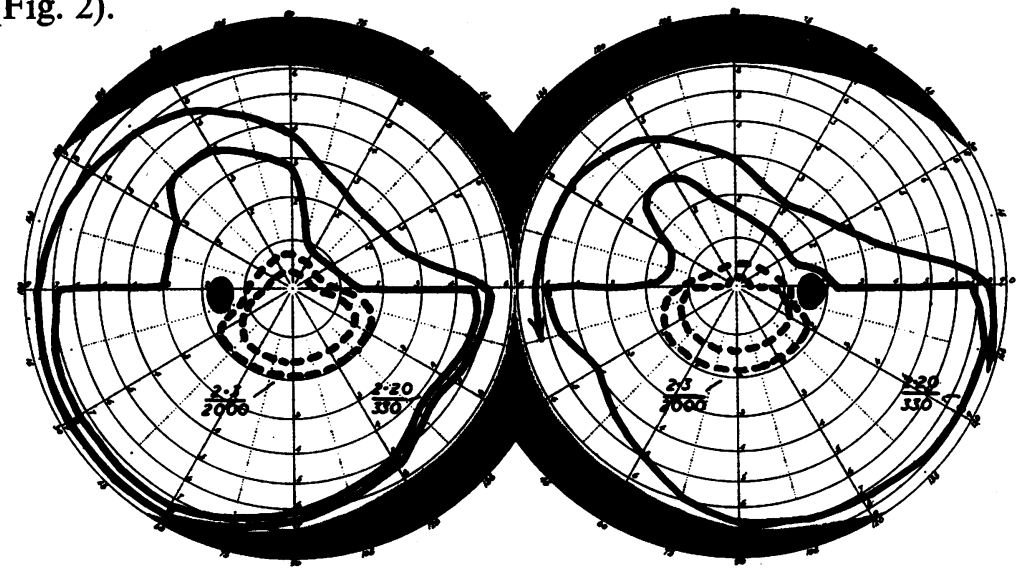

Fig. 2.-Visual fields in a case of arterial thromboses in both optic nerves associated with generalized arteriosclerosis. Gradual progression over 18 months.

Usually both eyes are affected, but occasionally the condition may appear in one eye some time before the other is affected. Many of these cases have peripheral field defects connected with the blind spot and suggestive of nerve fibre bundle defects. Conversely, isolated defects in the central field, or fibre bundle type, may be seen. These are difficult to distinguish from the fields of chronic glaucoma (Hughes, 1954; Fig. 47).

Additional evidence may be adduced from the indirect injuries of the optic nerve. The general consensus of opinion now favours a lesion in the optic canal whereby the nerve is displaced or twisted and small arterial twigs going to the pial plexus are torn. Inferior hemianopia is the commonest field defect and usually the macular area is spared as one would expect from the anatomy of this area. Fibre bundle defects and generalized depression are seen frequently and superior hemianopia occasionally (Hughes, 1945).

A great many lesions of the optic nerve produce field loss which has neither the features of horizontal hemianopia nor those of fibre bundle defects. Compression of the nerve is a particular example and has puzzled many generations of clinicians. Most writers are agreed that some form of central field loss is the commonest feature of compression whether the lesion be intracranial or orbital. I have found that a central scotoma with quadrantic features and often extension to the blind spot is so common as to be almost diagnostic of compression of the orbital portion of the nerve though less frequent with intracranial lesions (Hughes, 1954).

In most situations where compression takes place, the fibres subserving the central area of the field are placed in the centre of the nerve and the reason why they should be affected whilst peripheral fibres adjacent to the tumour are involved relatively late is a riddle as yet unsolved. The position with regard to the various theories has been admirably stated by Mooney and 
McConnell (1949). Meadows (1949) has suggested that such cases may be seen only in a late stage when break-through to the periphery has occurred and that even in the early case some peripheral loss may be present. This does not accord with my own experience and even when careful quantitative perimetry is done in the early case no peripheral defect can be detected though the central field changes themselves may be slight (Fig. 3).

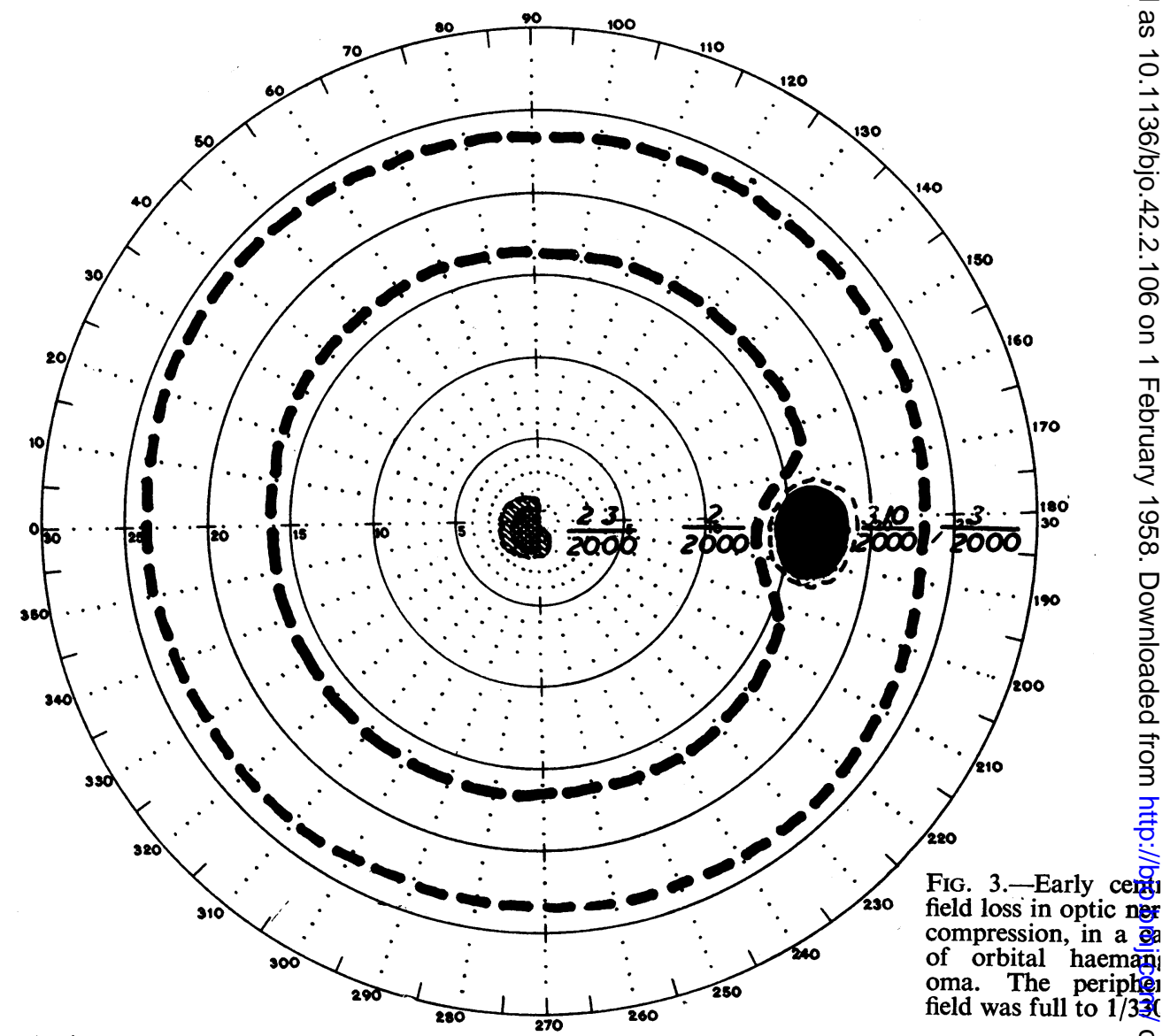

When peripheral involvement appears this is often along a horizontal meridian and not always related to the maximum area of the central scotoma, i.e. it is not a "break-through" (Fig. 4, opposite).

In very late stages of compression the whole of the central and intermediate field disappears, leaving only a rim of dim vision around the periphery, and this rim does not usually have hemianopic characteristics (Hughes, 1954; Fig. 95). It is difficult to explain these changes. Many writers have made a vague suggestion that "vascular factors" must play a part. Mooney and McConnell (1949) have stressed that vascular changes in the region of the optic canal produce predominantly peripheral field defects and 


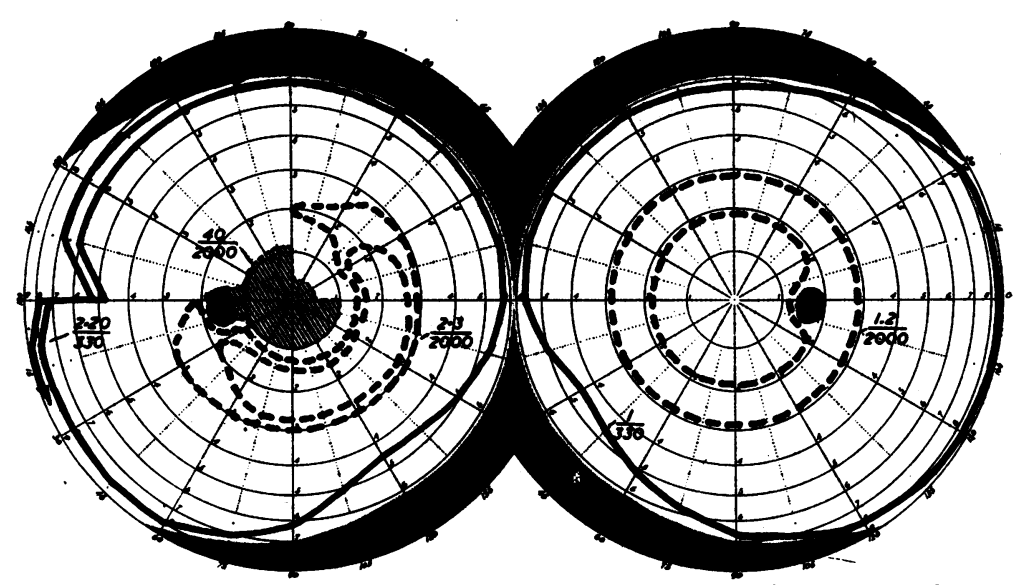

Fig. 4.-Early peripheral field loss in a case of optic nerve compression. Loss of central field is already far advanced before peripheral loss occurs and this is not related to break-through of a scotoma. From a case of "pseudo-tumour" of the orbit.

instance optic nerve injuries as an example of this. They have also made an interesting contribution in suggesting that central field loss is often associated with compressing lesions near the optic canal intracranially which may displace the nerve in relation to the canal and compress, secondarily, the ophthalmic artery. This hypothesis is amply supported in my own experience and may provide an explanation for the intracranial lesions at least. Definitive lesions of the ophthalmic artery producing central field loss have not, however, been reported and it is difficult to see why compression of an artery supplying all parts of the nerve should have this effect. The position with regard to intra-orbital lesions is more difficult. Here the common site for tumours is above the nerve, and the arterial supply to the central area lying beneath the nerve is cushioned from the tumour by the nerve itself and protected from counterpressure by soft tissues beneath. It is possible that downward and lateral pressure might nip the ophthalmic artery against the anterior edge of the optic canal and yet the type of field loss seems to be so constant wherever the tumour may be.

These field defects betray neither of the features associated with vascular lesions, horizontal hemianopias, and fibre bundle defects, and the bulk of evidence would suggest that the field loss is not of vascular origin but must be related to compression or kinking of the nerve fibres themselves.

\section{Part II. The Chiasma}

In the chiasma there is much greater agreement on the pattern of vascularization, at least as far as the central area is concerned; some disagreement still remains concerning the anterior chiasmal angle and intracranial portion of the optic nerves.

Earlier writers recognized a vascular network running upwards around the pituitary stalk from which many small branches ran to the chiasma itself. More recently writers have been concerned to map out individual supply $8^{*}$ 
branches and to determine their territory of supply. For instance, most early workers have described branches from all the vessels in the vicinity, including the ophthalmic, anterior cerebral, anterior communicating, and carotid artery, the hypophyseal arteries, middle cerebral and posterior communicating arteries, and the anterior choroidal artery. Several writers have admitted that the part played by some is a small one, and François and Neetens (1956) state that, during injection experiments, exclusion of the middle cerebral, posterior communicating, and anterior choroidal arteries resulted in non-filling of only a small area of the chiasma in the posterotemporal region. Nevertheless, they adhere to the original idea that the chiasma is supplied by eleven arteries, either directly or secondarily through a surface network of vessels.

Other writers, notably Steele and Blunt (1956) and Dawson (1948), have been more specific in their interpretation of this vascular network. They have described separate branches from the anterior cerebral and anterior communicating, from the carotid itself, and from the posterior communicating; in particular they cite the anterior superior hypophyseal artery from the carotid as being a major source of supply to the inferior surface of the chiasma.

One may, perhaps, justly combine the views so propounded in the following manner: On the inferior surface of the chiasma a major artery of supply is the anterior superior hypophyseal artery. This arises from the carotid, passes medially, and sends a major branch to the chiasma either directly or via the peri-infundibular plexus. It appears to supply much of the central region of the chiasma inferiorly and especially posteriorly and is claimed by Steele and Blunt to send forward a definitive branch to the under surface of the intracranial portion of the optic nerve which serves as its main supply. Branches also arise from the posterior communicating to supply (or at least enter) the postero-inferior angle of the chiasma (Fig. 5, opposite).

A separate branch arises from the medial surface of the internal carotid itself and enters the infero-lateral aspect of the chiasma. This is an important branch, for it is the only single artery of supply and it enters a region of the chiasma in which are situated the fibres from the uncrossed upper nasal field.

Most writers seem agreed that the superior aspect of the chiasma and its anterior angle are supplied by branches from the anterior cerebral and anterior communicating arteries. These arise from the anterior aspects of the vessels concerned and pass forward to enter the upper surface of the chiasma, some passing around the anterior margin. There is, however, some divergence of opinion concerning the vessels in this region. Dawson (1948) has described an anterior chiasmal plexus or arcade. This he states is composed of branches from the anterior cerebral and anterior communicating arteries, and is also largely contributed to by a vessel from the ophthalmic artery. 


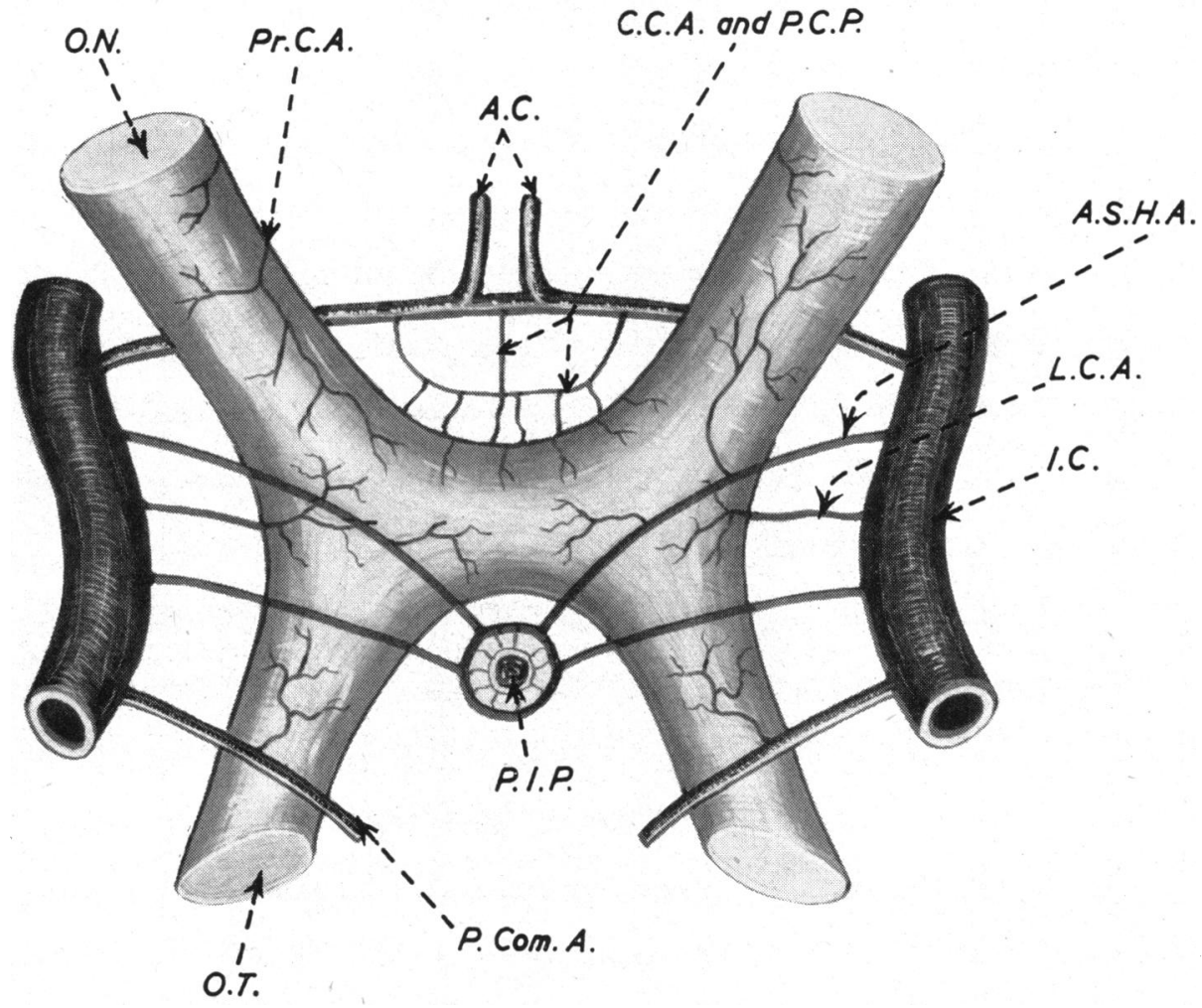

FIG. 5.-Schematic representation of the blood supply of the inferior surface of the chiasma. On the right side of the diagram the optic nerve is supplied by a twig from the anterior superior hypophyseal artery, on the left side by twigs from the ophthalmic artery (the prechiasmal artery of Dawson).

$\begin{array}{llll}\text { O.N. } & \text { Optic nerve } & \text { P.I.P. } & \text { Peri-infundibular plexus } \\ \text { O.T. } & \text { Optic tract } & \text { A.S.H.A. } & \text { Anterior superior hypophyseal artery } \\ \text { I.C. } & \text { Internal carotid artery } & \text { L.C.A. } & \text { Lateral chiasmal artery } \\ \text { A.C. } & \text { Anterior cerebral artery } & \text { C.C.A. } & \text { Central chiasmal artery } \\ \text { P.Com.A. } & \text { Posterior communicating artery } & \text { P.C.P. } & \text { Prechiasmal plexus }\end{array}$

This he describes as arising from the intracranial portion of this artery, running along the infero-medial aspect of the optic nerve and the anteroinferior margin of the chiasma to anastomose with its fellow of the opposite side and with the anterior cerebral branches. He names this the pre-chiasmal artery. Others, however, have failed to find this vessel in dissections. In fact, most writers state that the intracranial contribution of the ophthalmic artery is insignificant and perhaps confined to one or two small branches to the optic nerve near the canal. François and Neetens (1956) agree with the presence of an anterior chiasmal arcade, but have found it to be supplied by branches of the carotid, the anterior cerebral, and the anterior communicating; in addition branches of all these vessels might go direct to the chiasma. They found, in a third of their dissections, a specific artery from the anterior 
communicating which went direct to the chiasma; this they state to be the only definitive artery to the chiasma and, for this reason, they name it the chiasmal artery. On one occasion they were able to inject this vessel alone, and they found that its territory of supply was the central area of the chiasma, though filling in this area was incomplete.

All the workers cited are skilled and able observers who have without doubt described accurately the findings in their dissections. One can only conclude that the morphology of chiasmal arteries is very variable, at least as far as the anterior region is concerned, and that in most cases it is supplied from an arterial plexus but that in occasional cases definitive arteries may be seen: in particular the lateral branch from the carotid (the lateral chiasmal artery), the anterior branch from the anterior communicating (the central chiasmal artery), and possibly in rarer instances the prechiasmal artery from the ophthalmic.

Comparatively few writers have turned their attention to the arteriolar supply of the chiasma or to the capillary pattern, and amongst these François and Neetens (1956) and Dawson (1948) stand out. Surprisingly there seems to be agreement on the arteriolar pattern. This may be summarized as follows: Inferiorly branches enter the central area of the chiasma and tend to run posteriorly. Superiorly and anteriorly they tend to run posteromedially following the course of the crossing fibres. In addition two sets of arterioles are seen running in from the optic nerves. The lateral set run back postero-laterally in the direction of the uncrossed fibres whilst medially they run postero-medially following roughly the direction of the crossing fibres. Posteriorly arterioles enter from the region of the optic tract and run to the lateral area of the posterior chiasmal angle.

One may assume that the lateral fanning branches are derived from the lateral chiasmal artery, whilst the central and anterior crossing arterioles are derived from the anterior chiasmal area and central chiasmal artery. The inferior arterioles are presumably derived from the anterior superior hypophyseal artery. It is surprising that some arterioles are not seen running anteriorly towards the optic nerve if Steele and Blunt are correct in their descriptions.

It would be difficult to predict from this description what might be the result of a specific vascular occlusion, the arterial supply is in large part plexiform, and loss of a single branch might be expected to have little effect on the whole. Certain features of the anatomy are, however, suggestive. In the first place, the supply to the inferior uncrossed fibres seems to be by a definitive artery situated infero-laterally and well out of reach of the usual compressing tumour from the pituitary fossa. This may well explain the preservation of the upper nasal quadrant in so many advanced cases. Secondly, the supply to the post-inferior border, the site of the macular crossing, seems to be supplied from below, possibly by the superior hypophyseal artery or a branch from the posterior communicating. Lesions in 
this area, therefore, might be expected to produce early central hemianopic scotomata. If we accept the presence of a central chiasmal artery in some cases, then a small lesion anteriorly might well produce marked bitemporal loss without gross evidence of pressure. Again the occasional finding of marked bitemporal loss (as well as optic nerve defects on one side) in laterally placed lesions under the optic nerve might be explained by the presence, in that instance, of an ophthalmic branch, the pre-chiasmal artery of. Dawson. Generally speaking, however, it will be seen that the arterial supply is closely related to the fibres it supplies, so that in a given instance it is hardly conceivable that one could separate the effects of direct pressure on fibres from those of occlusion of small arterioles.

The capillary supply seems to have relatively little importance. When viewing the chiasma in low magnification stained for vessels, one is struck by the apparent similarity between the fibre patterns and the vascular pattern.

Lateral capillaries run roughly antero-posteriorly as do the uncrossed fibres, whilst central capillaries, especially those anteriorly placed, sweep across the midline in just the way of the crossing fibres. The excellent work of François and his colleagues has shown that this is hardly the case, and they have divided the chiasma into no less than six paired zones, and one central zone, in which the capillary formation, including size of vessels, density, and method of branching, are all distinct.

One's first view of the chiasmal capillary network is probably, therefore, misleading. In particular, it is noteworthy that, though the central capillaries sweep across in a superficial resemblance to the crossing fibres, they do not intermingle or criss-cross in the centre as do the fibres. In addition, there is no evidence in the capillary network of the fibre loops in the optic nerve and tract so clearly described by writers on fibre anatomy.

Study of vascular pattern in the supero-inferior direction is also disapointing, for one so often sees in visual field loss a quadrantic element to a defect suggesting that there may be a vascular raphe corresponding to the horizontal meridian in the chiasma as well as the retina and optic nerve. No evidence of such a raphe has been found by me or by other writers on the subject.

Comparatively little attention has been paid to the venous picture in writings on the vascularization of the nervous system, though, as most of us well know, the effects of venous thrombosis or obstruction may be as lethal to neural function as arterial obstruction. According to Dawson (1948), venous plexuses are present above and below. Superiorly, the veins run upwards from the plexus to join the anterior cerebral veins running with the anterior cerebral arteries. Inferiorly, the veins run posteriorly and join a pre-infundibular vein or venous plexus from which a large vein runs posterolaterally on either side to join, eventually, the basal vein of Rosenthal. Really very little seems to be known of the territory of drainage of these vessels and further study might well repay the time spent on a difficult technique. 
It might be useful now to approach the subject from another angle and examine the clinical experience of visual field loss to ascertain whether there are any specific features which can be ascribed to a vascular disturbance.

In the majority of instances field loss is associated with gross disturbance of anatomy by tumour or other process and the usual reaction is one of surprise that the disturbance of function is not much greater than it is. As has been suggested above, arteries and veins are mostly closely related to the fibres they supply and it is impossible in a given instance to separate the effects of direct compression, arterial and venous obstruction. There is considerable difference of opinion on the role of vascular obstruction, for François and Neetens (1956) have stated that in their view field loss is nearly always due to the mechanical effects of compression rather than to vascular obstruction, whereas Harrington (1956) believes that vascular obstruction is the major cause of field loss in cases of brain tumour. It may, therefore, prove fruitful to examine certain categories of field loss to see what light they may throw on the vascularization of the optic pathways or, in other circumstances, whether a knowledge of the anatomy of vascular supply can explain apparently incongruous clinical findings. Obviously it will be worthwhile to examine field defects presumed to be due to disease of the vessels themselves either from thrombosis, spasm, or the effects of trauma. Some cases in which the field loss appears to implicate fibre tracts at a distance from the causative lesion may well repay study also, and, lastly, those cases in which the pattern of field loss, or more often preservation, are difficult to explain on purely mechanical grounds.

Instances of direct involvement of chiasmal vessels by arterial disease are rare in the literature. The clinical interpretation in such cases is confused by many factors, not the least of which may be individual convictions. The role of arterial obliteration, primary neural degeneration, arachnoiditis, and compression from calcified plaques in vessel walls all have to be sorted out, and in the majority of cases to ascribe a given field defect to one particular factor proves to be impossible, even with the aid of histological examination. In the few cases I have seen and have felt reasonably satisfied that arterial obliteration was the primary factor the field loss has been a simple bitemporal depression affecting the upper quadrants principally (Fig. 6, opposite).

In this case the subject was an elderly man with general arteriosclerosis, the mode of onset extremely sudden and followed by a period of improvement over some weeks. In fact, the clinical features, general condition of the patient, and subsequent history were all suggestive of a small arterial thrombosis. I have since seen several such cases and, whilst the pathological confirmation is lacking in them all, this clinical experience suggests that there is some definitive arterial supply to the antero-inferior region of the chiasma. The work described above would tend to support this view and the anatomical and clinical material taken together support the hypothesis that there may be, at least in some cases, a definitive artery of supply to this area of the chiasma. 


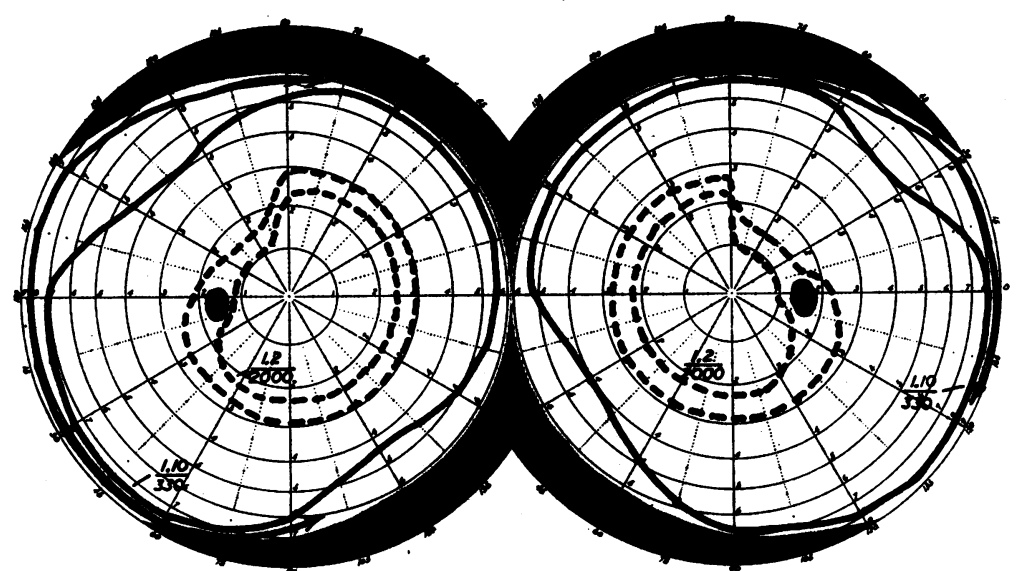

Fig. 6.-Bitemporal depression in arteriosclerosis of chiasmal vessels. Sudden onset in a man of 74 with some improvement during the next 6 weeks.

Whether this is the chiasmal artery of François and others derived from the anterior communicating, or the pre-chiasmal artery of Dawson derived from the ophthalmic, there would seem to be some divergence of opinion. Other views have been expressed, however, as to the characteristic visual field loss in arteriopathic lesions, and Traquair (1948) states that arterial obstruction and small softenings in the chiasma may be responsible for bitemporal hemianopic central scotomata with normal peripheral fields. This work suggests that other areas of the chiasma may have a definitive arterial supply. Each individual has only a small experience of such cases and it seems likely that only the pooling of experience and especially of pathological material from many sources is likely to advance our knowledge on a sound basis.

The mechanism of indirect chiasmal injury remains somewhat of a mystery. In reported cases operative inspection has not demonstrated any antero-posterior tearing such as one might expect from the clinical picture (Hughes, 1945), though such, indeed, has been described (Rand, 1936). Traquair, Dott, and Russell (1935) were the first to suggest that chiasmal dysfunction might be accounted for by damage to small arteries and subsequent thrombosis. In most cases the field loss is bitemporal and complete, though where some visual field is spared it is usually in the lower quadrant and might compare with the arteriopathic cases described above. In some personal cases there has been sparing of central temporal vision and this would suggest that damage has been confined to the territory of the chiasmal or pre-chiasmal artery, but perhaps this is special pleading and as far as I know there is no histological evidence available on this point.

In a case previously reported (Hughes, 1943), there was apparent injury to the anterior chiasmal angle. In the affected eye only an upper nasal field remained; in the opposite eye the upper temporal quadrant was depressed. I have since seen several such cases and in all but one the optic nerves and 
chiasma appeared to be normal at operative inspection. In the single abnormal case the anterior chiasmal angle appeared grey and soft and had the macroscopic appearances of infarction. Here, unfortunately, histological confirmation is lacking and the lesion may have been simply a local contusion.

Pressure on the chiasma from tumour and other causes provides frequent examples of field defects difficult to explain on the purely mechanical basis of compression. Noteworthy amongst these is the apparent immunity of the upper nasal quadrant of the field. Even in very advanced cases of compression, when the whole of the temporal fields, lower nasal fields, and macular area have been lost, a small upper nasal field quadrant may remain intact for years (Fig. 7). Most chiasmal compressions are the result of compression from below and anteriorly by pituitary adenomata and the sequence of field loss is well recognized: upper temporal, lower temporal, and then lower nasal (Traquair, 1948; Hughes, 1954). Yet survey of the anatomy would suggest in theory that spread should be expected from the temporal field to the upper nasal, for the lower lateral fibres are those most closely associated with the tumour, and the tendency for the optic nerves to be rotated laterally would bring these fibres into even closer contact with it. Many have commented on this phenomenon and most are agreed with the sequence of events, though Traquair (1948) states that occasionally the upper nasal quadrant may be affected at a relatively early stage and quotes a case of Hirsch in which there was retention to a late stage of small symmetrical fields in the lower nasal quadrant.

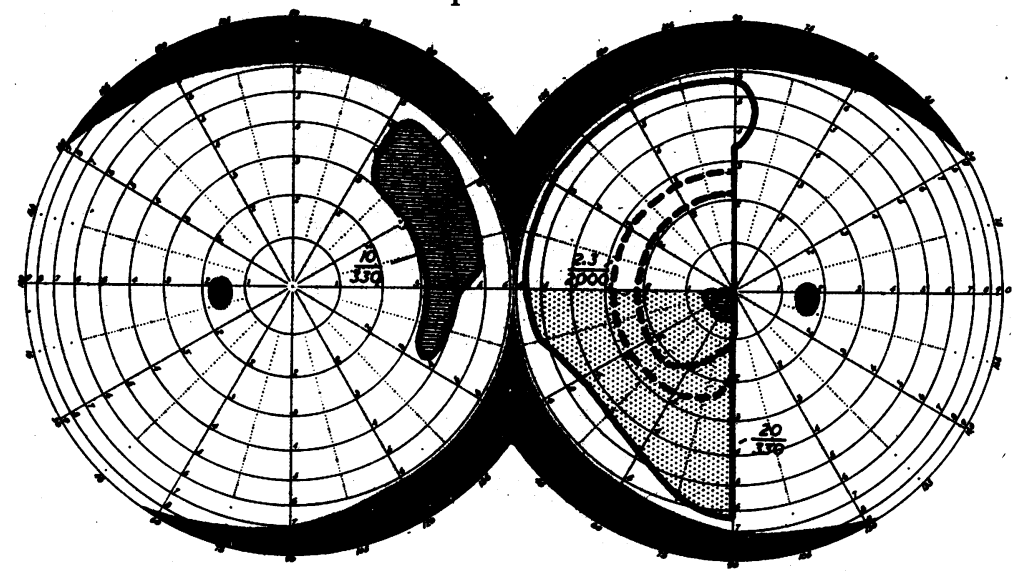

Fig. 7.- Residual upper nasal island of vision on the left in a case of chromophobe adenoma of the pituitary.

Many explanations of this field defect have been put forward, but none is wholly satisfactory. That the nasal field per se should be affected late is not difficult to understand. The lateral fibres are far removed from tumour pressure and only held against the tumour by the checking action of crossing medial fibres. As soon as these have been destroyed the lateral chiasmal tracts can easily be separated and can accommodate themselves to the growing tumour. Such a sequence of events is often reflected in an apparent 
halt in the progress of a field defect after the temporal fields have been lost. Why the upper nasal field should be affected after the lower and often remains invulnerable for so long a time is much more difficult to explain. Traquair (1948) suggests that the upper fibres lie more medially in the lateral region of the chiasma and may so come to be affected before the lower fibres. Even if this explains the sequence of field loss, it offers no reasonable solution of the problem of the invulnerable upper nasal quadrant.

Other writers, notably Rucker and Kernohan (1954), have implicated the anterior cerebral arteries and have suggested that counter pressure of these vessels on the upper surface of the optic nerves or chiasma, often with deep grooving, may explain the lower nasal field loss. Much interest has been taken recently in this concept though the condition was described over a hundred years ago (Turck, 1852). Operative inspection of a large number of pituitary tumours has led me to believe that this factor plays rather a small part in the development of field loss. In many of the cases reported the lower nasal field defect has occurred early and is present even when quite large areas of the temporal field are retained. As an explanation of combined lower nasal and temporal field loss in an early stage of pituitary tumour compression, I feel it is quite adequate, but I doubt whether it is an explanation of the usual orderly sequence of field loss in these tumours. In one example a deep groove was present on the optic nerve but there was no associated field loss (Fig. 8).

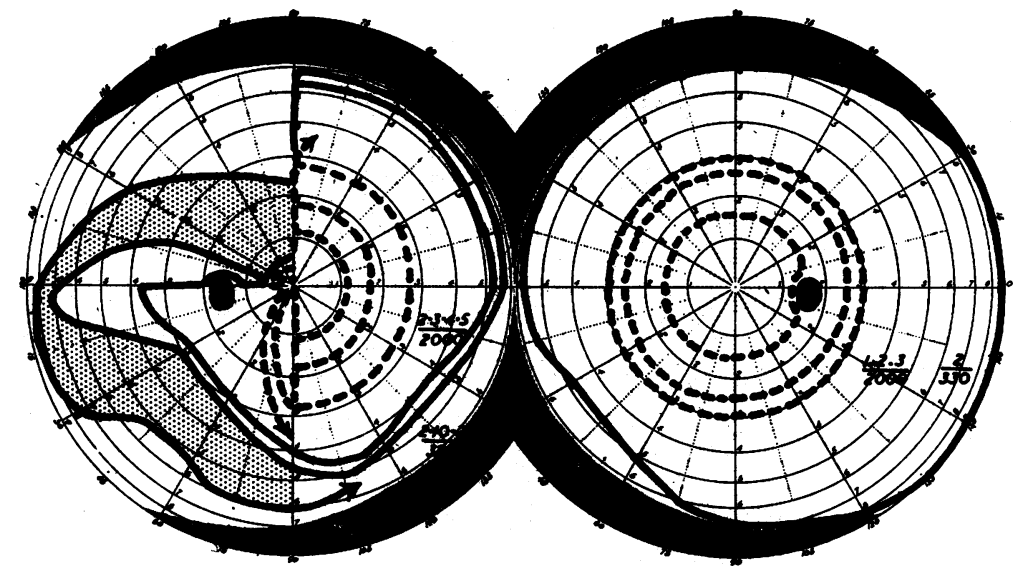

FIG. 8.-Chromophobe adenoma of pituitary affecting chiefly the left optic nerve. Operation disclosed a deep groove on the right side of the chiasma, but the visual field in the right eye had been normal only 12 hours before operation.

Other writers have suggested that the phenomenon is due to displacement of the optic nerves against the firm walls of the optic canal. This may well explain some cases of nasal field loss and, if the nerves were to be rotated laterally as suggested, then pressure from this cause might well fall on the upper lateral fibres. - I would still doubt that this is the "normal" course of events, though it may well, like the previous example, explain the early 
lower nasal field loss seen in some cases. Operative inspection of the nerves and chiasma in these cases has revealed a fairly common phenomenon. The crossing fibres in the chiasma are grey, fragmented, and hidden behind the mass of the tumour, the medial aspects of the optic nerves have the same, grey, avascular appearance. At the junction of the chiasma and lateral uncrossed fibres, there is a triangular bluish-grey area. This appearance is given by thinning of fibres in this region and in some instances tumour may be seen protruding through this area. The lateral bundles, presumably comprising the uncrossed fibres, appear normal, pink, and well-vascularized. This presumably, is the early stage of separation of crossed from uncrossed fibres by the tumour. Yet the explanation cannot be entirely mechanical for much of the grey area does not appear to be directly compressed by the tumour. The appearances may well be produced by infarction of this area leaving the lateral uncrossed fibres well-vascularized. Turning now to the anatomy of chiasmal vascularization, an important contribution may well be that of Dawson (1948) who, after many careful dissections, came to the conclusion that a branch from the carotid artery to the lateral and inferior aspect of the chiasma was the only definitive chiasmal artery he could identify. In just such a place we would expect to find the inferior uncrossed fibres from the upper nasal field. The fact that these fibres may have a definitive blood supply coming from a lateral aspect where it would be least subject to pressure from a midline tumour may well be the usual explanation of the preserved upper nasal quadrant.

In a few cases one sees an apparent chiasmal compression in which the upper nasal quadrant has been lost before the lower. In most of such cases in my experience the compression has been primarily of the optic nerve. A little further support for this thesis is obtained from consideration of lesions compressing the chiasma from the lateral aspect. In such instances the upper nasal quadrant is not especially preserved and in fact some cases suggest that it may be more vulnerable. Such evidence, however, is not particularly helpful, for in these cases the fibres concerned are subject to the direct effects of compression.

From consideration of compression fields, therefore, one would suspect that the fibres concerned with the upper nasal peripheral field are especially exempt from the effects of pressure, whilst the lower nasal and the whole of the temporal and macular areas are affected by pressure in the way one would expect. One can only conclude by suggesting that these fibres have some individual source of blood supply unaffected by the tumour.

It is common to find sharp quadrantic features in chiasmal field loss. It is very frequent in the macular field and often initial lower nasal loss in this region is of this character, the line of demarcation running exactly along the horizontal meridian (Fig. 9, opposite). This quadrantic loss is less often seen in the peripheral field, but none the less does occur and often, when greying is present, the line of demarcation runs exactly along the horizontal meridian.

Study of the fibre arrangement in the chiasma shows no evidence at all of 


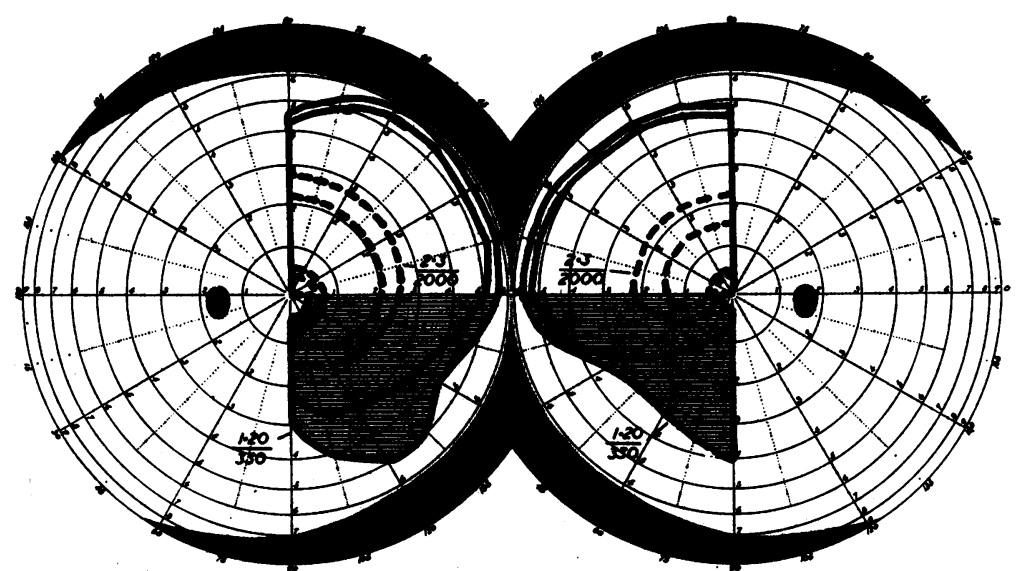

Fig. 9.- "Quadrantic" features in chiasmal compression, from a case of chromophobe adenoma of the pituitary.

any such quadrantic pattern and it is inconceivable, looking at sections of the chiasma, that this type of field loss could be due simply to the mechanical effects of pressure on such an inextricable tangle of fibres. Such quadrantic features in field loss are common in lesions of the optic nerve, especially with regard to the horizontal meridian, and are usually ascribed to "vascular" lesions. It may be that in such cases this is the effect of optic nerve compression, but in many instances the field loss has such clear chiasmal characteristics, namely, the clear-cut limitation by the vertical meridian. There is no evidence available so far as to supero-inferior fields of blood supply and, in fact, François and Neetens (1956) could find no characteristic features of vascularization in the supero-inferior plane. Be this as it may, the existence of two separate sources of supply, superior from the anterior cerebral and anterior communicating and inferior from the carotid, hypophyseal, and posterior communicating, may have some significance. One must, however, consider this problem as so far largely unsolved.

Compression of the chiasma produces scotomata of various kinds in the visual fields. They may be central and quadrantic, ring-like, circular, or peripheral, and may occur in any part of the field, though the central area is most common; scotomata extending into the nasal field are very rare and are probably due to super-added optic nerve compression. Those in the temporal field have one feature in common in that they never pass the vertical meridian, and this strongly suggests their chiasmal origin. Amongst these scotomata one encounters some which bear a strong resemblance to the nerve fibre bundle scotomata seen in optic nerve lesions (Fig. 10, overleaf). They mostly arise from the area of the blind spot though more central arcuate scotomata may be seen. I have not yet encountered a sector defect in the temporal field such as is seen in the optic nerve and retinal lesions. The occurrence of such scotomata is strongly suggestive of an individual and definitive blood supply to bundles of fibres just like that seen in the optic nerve, and one may presume that this fibre bundle system is continued for 


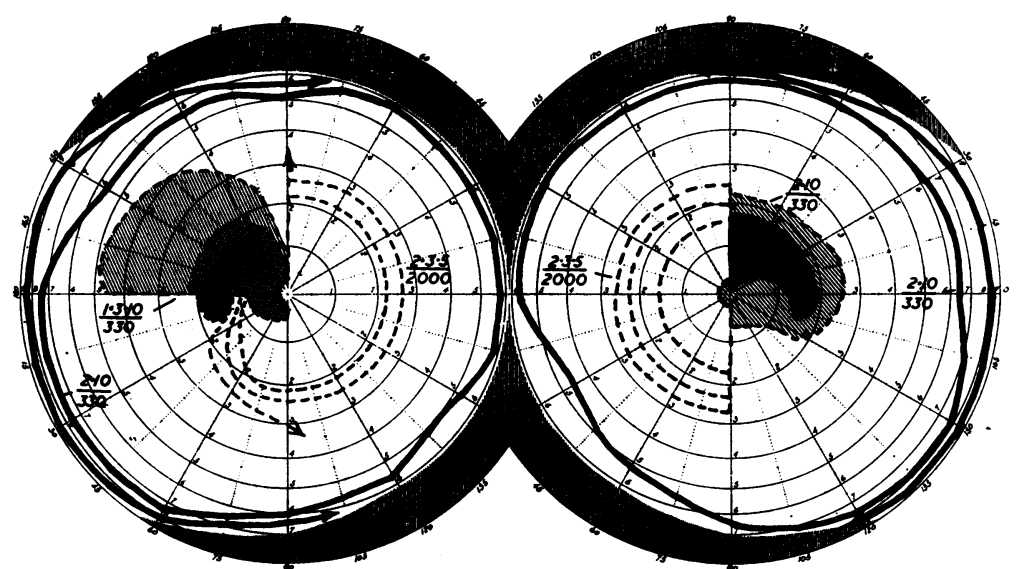

Fig. 10.-Nerve fibre bundle scotomata in chiasmal compression, from a case of chromophobe adenoma of the pituitary.

some way at least into the chiasma (Dubois-Poulsen, 1952). Most observers have noted, however, that the arteriolar and capillary pattern does not follow the fibre pattern in the chiasma. Most of such scotomata are in the central temporal field and yet the vascular pattern of the macular fibre area is as haphazard as anywhere in the chiasma. In my experience, most of such examples have been in patients with post-fixed chiasmata and long optic nerves, and this may well be a sign of such a variant, the field loss being in reality related to the anterior chiasmal angle in which the fibre bundle arrangement and vascular pattern is prolonged from the optic nerves.

Lastly, there is one aspect of chiasmal compression fields that is very difficult indeed to explain; the residual temporal island. In a small proportion of fields, usually those of a scotomatous character, the initial central scotoma breaks through to the upper and lower temporal periphery, isolating a small island of vision far out in the temporal field (Fig. 11). This

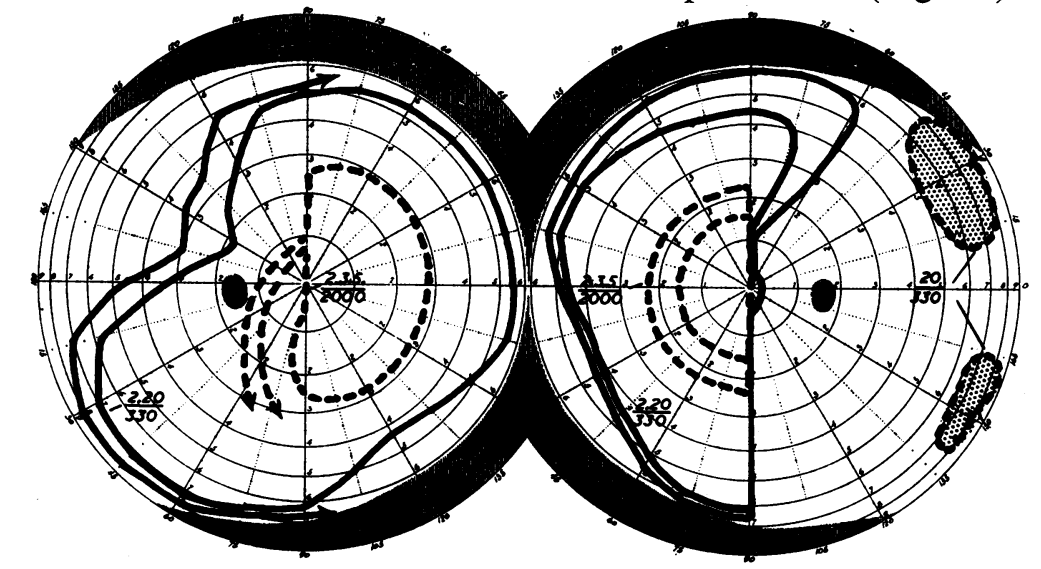

FIG. 11.-Temporal islands in chiasmal compression, from a case of chromophobe adenoma of the pituitary. 
island tends to persist and may still be present long after the remainder of the temporal field has vanished and the nasal field become involved. These islands most often occur above or below the horizontal meridian and may represent the forward and backward loops of peripheral fibres in the chiasma. Traquair (1948) and Dubois-Poulsen (1952) both state that such islands are not seen when the lesion is prechiasmal or affecting the inner sides of the optic nerves, and yet examples following trauma have been reported. The only conceivable explanation is that the looping fibres are protected by their position from lesions affecting the antero-central area of the chiasma as far as the inferior field is concerned and the postero-central area as far as the upper field is concerned. Whether in these situations such fibres also enjoy a selective blood supply with the uncrossed fibres amongst which they lie is purely speculative. On the other hand such islands often cross the horizontal meridian and as such one would have to look for a situation in which the upper and lower peripheral fibres lie together. In the body of the chiasma itself, if we are to believe present concepts as to fibre anatomy, they lie as far apart as it is possible. The lesions in such cases, therefore, can only be in the exact centre of the chiasma.

\section{REFERENCES}

Dawson, B. H. (1948). M.D. Thesis, Manchester.

Dubois-Poulsen, A. (1952). "Le champ visuel". Masson, Paris.

FrançoIs, J., and NeEtens, A. (1954). Brit. J. Ophthal., 38, 472.

$\longrightarrow$ ( $\longrightarrow$ (1956). Ibid., 40, 45. (1955). Ibid., 39, 220.

HALlER, A. voN (1754). "Arterium oculi historia et tabulae arterium oculi". Gottingen.

HARRINGTON, D. O. (1956). " "The Visual Fields". Kimpton, London.

HugHes, E. B. C. (1943). Brit. J. Ophthal., 27, 367.

(1945). In a discussion on the ocular sequelae of head iniuries, Trans. ophthal. Soc. U.K., 65,35 .

Hughes, B. (1949). Ibid., 69, 411. (1954). "The Visual Fields". Blackwell, Oxford.

Meadows, S. P. (1949). Proc. roy. Soc. Med., 42, 1017.

Mooney, A. J., and McConnell, A. A. (1949). J. Neurol. Neurosurg. Psychiat., 12, 205.

RAND, C. W. (1936). Arch. Surg., 32, 945.

RuCKer, C. W., and Kernohan, J. W. (1954). A.M.A. Arch. Ophthal., 51, 161.

Steele, E. J., and Blunt, M. J. (1956). J. Anat. (Lond.), 90, 486.

Traquair, H. M. (1948). "An Introduction to Clinical Perimetry", 5th ed. Kimpton, London. , DotT, N. M., and Russell. W. R. (1935). Brain, 58, 398 .

Turck, L. (1852). Z. Ges. Aertz. Wien, 8, 299. Quoted by Rucker and Kernohan (1954).

Vossius, A. (1883). v. Graefes Arch. Ophthal., 29, pt 4, p. 119.

WolfF, E. (1948). "The Anatomy of the Eye and Orbit", 3rd ed. Lewis, London.

WYBAR, K. C. (1956). Brit.J. Ophthal., 40, 65-81.

ZINN, J. G. (1755). "Descriptio anatomica oculi humani”. Vandenhoeck, Gottingen. 